\title{
Germanica
}

\section{Contrefaçons de fictions policières}

Nachgemachte Kriminalliteratur

Jean-Jacques Pollet

\section{(2) OpenEdition}

Journals

Édition électronique

URL : http://journals.openedition.org/germanica/2178

DOI : 10.4000/germanica. 2178

ISSN : 2107-0784

\section{Éditeur}

Université de Lille

\section{Édition imprimée}

Date de publication : 31 décembre 1993

Pagination : 163-178

ISSN : 0984-2632

\section{Référence électronique}

Jean-Jacques Pollet, «Contrefaçons de fictions policières », Germanica [En ligne], 13 | 1993, mis en ligne le 10 février 2014, consulté le 06 octobre 2020. URL : http://journals.openedition.org/germanica/ 2178 ; DOl : https://doi.org/10.4000/germanica.2178

Ce document a été généré automatiquement le 6 octobre 2020.

(C) Tous droits réservés 


\title{
Contrefaçons de fictions policières
}

\author{
Nachgemachte Kriminalliteratur
}

Jean-Jacques Pollet

1 Soient quelques titres, évidemment choisis avec préméditation, correspondant à une sélection non limitative, à fin démonstrative: Der Hausierer (1967), Die Angst des Tormanns beim Elfmeter (1970), de Peter Handke; Schattengrenze (1969), Einladung an alle (1972), de Dieter Wellershoff; Albissers Grund (1974), d'Adolf Muschg; Die verlorene Ehre der Katharina Blum (1974), de Heinrich Boll ; Das Versprechen (1958), Justiz (1985) de Friedrich Dürrenmatt ; Blaubart (1982), de Max Frisch ; Eine gefährliche Begegnung (1985), d'Ernst Jünger... Leur point commun: à propos de chacun d'entre eux, aussi dissemblables soient-ils les uns des autres, la critique a évoqué, pour les décrire, une contrefaçon de fiction policière. Au-delà de cette évaluation «extérieure ", confirmée d'ailleurs dans la majorité des cas par les propos des auteurs - interviews ou commentaires-, il apparaît surtout décisif que cette référence soit inscrite explicitement dans les textes eux-mêmes, comme si elle était nécessaire au fonctionnement et à la lecture de ceux-ci.

2 L'objet de la présente analyse est d'essayer de repérer quelques-uns des modes de transactions entre littérature consacrée et fiction policière, qui semblent particulièrement productives pour le roman allemand contemporain.

\section{Mimesis policières?}

3 Pour cerner les données du problème, il nous paraît éclairant, en préambule à la lecture des œuvres, de rapprocher quelques réflexions "théoriques", dues aux auteurs concernés. L'histoire-cadre de Das Versprechen fournit comme on sait à F. Dürrenmatt le prétexte d'un exposé détaillé sur la narratologie policière à travers le dialogue entre le commandant H., ancien chef de la police cantonale de Zurich, et le narrateur, lui-même auteur de romans policiers :

[...] um ehrlich zu sein, ich habe nie viel von Kriminalromanen gehalten und bedaure, dass auch Sie sich damit abgeben [...]. Ich ärgere mich über die Handlung in euren Romanen. Hier wird der Schwindel zu toll und zu unverschämt. Ihr baut 
eure Handlungen logisch auf; wie bei einem Schachspiel geht es zu, hier der Verbrecher, hier das Opfer, hier der Mitwisser, hier der Nutzniesser; es genügt, dass der Detektiv die Regeln kennt und schon hat er den Verbrecher gestellt [...]. Diese Fiktion macht mich wütend. Der Wirklichkeit ist mit Logik nur zum Teil beizukommen [...]. Die Wahrheit wird seit jeher von euch Schriftstellern den dramaturgischen Regeln zum Frasse hingeworfen [...]. Ihr versteht nicht, euch mit einer Realität herumzuschlagen, die sich uns immer wieder entrieht, sondern ihr stellt eine Welt auf, die zu bewältigen ist. Diese Welt mag vollkommen sein, möglich, aber sie ist eine Lüge ${ }^{1}$.

4 La "vérité » contre l'affabulation frauduleuse : il est reproché fondamentalement à la fiction policière de n'être pas «réaliste». Curieusement, le narrateur, censé représenter la corporation des auteurs policiers (avec lesquels Dürrenmatt lui-même s'est précédemment commis, en publiant Der Richter und sein Henker et Der Verdacht) ne réplique pas aux arguments de son interlocuteur. Il aurait pu rétorquer qu'on faisait là un faux procès, en ce sens où la littérature policière, tout au moins celle dite de « détection » (et c'est bien à cette catégorie, visiblement, que se réfère le commandant H.) n'a jamais véritablement affiché de prétention «mimétique ». Que l'on relise le code poétique de Van Dine : la règle $n^{\circ} 16$ recommande explicitement " d'éviter les longs passages descriptifs, les analyses subtiles de caractère, les préoccupations d'atmosphère, qui ne peuvent qu'encombrer lorsqu'il s'agit d'exposer clairement un crime et de chercher le coupable $»^{2}$. C'est d'ailleurs ce trait non-descriptif qui a suscité, comme on sait, l'intérêt de Bertold Brecht, qui retient en priorité ce qu'il appelle «l'exercice intellectuel sur les lois de la causalité ${ }^{3}$, par lequel la littérature policière rejoindrait d'ailleurs les préoccupations du théâtre épique. Helmut Heissenbüttel, dans son essai Spielregeln des Krimilnalromans, conteste quant à lui le "réalisme » non seulement du récit de détection, mais également $d u$ "thriller» : dans les deux cas, les éléments en charge d'une éventuelle fonction mimétique - comme la topographie font l'objet d'une "stylisation " qui les constitue non pas en une image de la réalité, mais en un «modèle exemplaire " . Dürrenmatt est trop avisé pour ne pas savoir qu'il est donc paradoxal d'interroger la fiction policière sur son prétendu « réalisme ». C'est tout le pari de son récit, auquel il donne le sous-titre de «Requiem pour le roman policier » : le genre n'est pas abandonné, mais subverti ; Dürrenmatt lui demande, sous réserve d'aménagement - il avoue dans sa postface " une critique du personnage du détective, l'une des figures littéraires les plus typiques du dix-neuvième siècle »- de fournir, même si cela n'appartient pas à sa vocation et lui est peut-être même contraire, une représentation de la réalité. Un réalisme en quelque sorte " par défaut ».

Evidemment sur d'autres bases, Peter Handke développe une réflexion au moins comparable dans sa démarche, à propos de son roman Der Hausierer :

Was gibt eine Darstellungsweise, die schon längst kanonisiert ist, noch her für eine Darstellung der Wirklichkeit, meiner Wirklichkeit? Ich habe also das Kriminalromanschema nach «mir» abgesucht, und umgekehrt habe ich «mich» abgesucht, was von mir schon so weit literarisch durch Schemata bestimmt war, dass es dem Kriminalroman entsprach. Indem ich das Schema prüfte, es abstrahierte, mir seine unbewussten Regeln bewusst machte, hoffte ich, auch zu neuen Darstellungs- Möglichkeiten meiner zu kommen [...]. Ich wollte ein Darstellungsschema aus der Tivialliteratur wieder «wirklich» machen und neue Möglichkeiten auch den Lesenden zeigen : neue Möglichkeiten zu lesen, zu spielen, zu überlegen: zu leben ${ }^{5}$.

6 Le déplacement d'accent, par rapport à Dürrenmatt, est tout entier dans le possessif : voici un mode de représentation de «ma réalité » - une restriction qui convient tout à 
fait à l'écrivain «habitant de la tour d'ivoire " (Ich bin ein Bewohner des Elfenbeinturms). On notera également le renversement de perspective: l'écrivain ne se propose pas d'appréhender la réalité à travers les conventions (remaniées) du genre littéraire, mais interroge ces conventions sur la «réalité» (subjective) qu'elle peuvent articuler. Autrement dit: Peter Handke ne connaît pas d'avance ce qu'il cherche, n'a (apparemment, à ce qu'il prétend tout au moins) aucune idée préconçue de la "réalité » à découvrir grâce aux poncifs de la fiction policière - tandis que Dürrenmatt laisse clairement deviner son « a priori », l'ordre théologique du monde. Le seul « a priori »mais c'en est encore un - serait, chez Handke, le primat de la littérature.

7 L'argumentation de Dieter Wellershoff se rapproche de celle de P. Handke en ce sens où il part lui aussi d'une réflexion sur la forme de la fiction policière, qu'il assimile comme P. Handke à la littérature de consommation («Trivialliteratur») ${ }^{6}$. Il entend par là une littérature « lénifiante » («Beruhigungsliteratur»), dans la mesure où elle vient toujours confirmer le lecteur, après l'avoir fait provisoirement trembler, dans sa perception routinière et superficielle de la réalité, à laquelle il oppose la «vraie» littérature, fondamentalement « irritante » :

Sie versucht den Leser zu irritieren, ihm die Sicherheit seiner Vorurteile und gewohnten Handlungsweisen zu nehmen, sie macht ihm das scheinbar Bekannte unvertraut, das eindeutige vieldeutig, das Unbewusste bewusst und öffnet ihm so neue Erfahrungsmöglichkeiten, die vielleicht verwirrend und erschreckend sind, aber auch die Enge und Abstraktheit der Routine durchbrechen, auf die er in seiner alltäglichen Praxis angewiesen bleibt ${ }^{7}$.

D. Wellershoff ne fait même pas d'exception, à l'intérieur du genre policier, pour le roman noir américain des années 30, les «hard-core-thrillers» d'un Raymond Chandler ou Dashiell Hammet ; certes, le personnage du détective est sorti de l'intérieur cossu de Baker Street pour plonger dans la grande ville sordide, mais le « privé » n'a au fond pas plus de « réalité » que Sherlock Holmes, d'autres clichés se sont simplement substitués aux anciens. Il appartient maintenant au romancier de savoir user, le cas échéant, des conventions de la littérature policière, de les détourner de leur fonction première, afin qu'elles servent à " conserver ou reconstruire l'étrangeté du monde, à empêcher sa banalisation $»^{8}$.

9 Il nous semble que D. Wellershoff, au plan de la réflexion poétique, vient ici occuper une position intermédiaire entre F. Dürrenmatt et P. Handke. Il souscrit sans doute au principe handkéen du primat de la forme - il convient d'essayer, de mettre à l'épreuve une certaine structure narrative pour découvrir l'éventuelle « réalité » qu'elle articule et non pas, inversement, de chercher, à partir de la réalité soi-disant donnée, la structure qui l'exprime de manière la moins inadéquate. Mais il ne s'enferme pas pour autant dans le solipsisme - le monde existe et a un sens, tout au moins une virtualité de sens, en dehors du sujet lui-même. Dire que le monde doit être rendu dans son "étrangeté », c'est encore admettre, certes de manière minimale, une "Weltanschauung», même si celle-ci ne se confond pas, naturellement, avec la vision théologique d'un Dürrenmatt. Schématisons: une certaine contrefaçon de fiction policière est mobilisée chez $\mathrm{F}$. Dürrenmatt pour dire le monde, chez $\mathrm{P}$. Handke pour se dire soi-même, chez D. Wellershoff pour dire certaine relation au monde. Quelle que soit la stratégie particulière dans laquelle elle s'inscrit, on retiendra que la subversion de ce code narratif s'opère dans tous les cas au profit d'un (nouveau) "réalisme », perçu (certainement au prix de simplifications) comme étranger à l'orthodoxie et/ou à la tradition du genre. 
10 Afin de décrire, maintenant, quelques-uns de ces modes de subversion «à l'œuvre ", il nous paraît judicieux de reprendre la distinction classique, théorisée (entre autres) par R. Alewyn, entre "récit de détection » (Detektivgeschichte) et " histoire criminelle » (Kriminalerzählung) 9 .

\section{Enquêtes dérisoires}

11 Rappelons les caractéristiques principales du modèle narratif du récit de détection, sans entrer dans le détail de ses multiples variantes:

1) Point de départ de l'intrigue, l'événement du crime, affecté d'une ou de plusieurs inconnues : qui ? Comment ? Pourquoi?

2) Acteur principal, qui focalise l'ensemble de la narration: le détective (ou ses substituts).

3) Moteur de l'intrigue, les différentes étapes de l'enquête, fondée sur la collection des indices, ce qui implique une relation " rétrospective ».

4) La conclusion du récit désigne un coupable, permettant ainsi une restauration du droit de la raison.

Le roman de F. Dürrenmatt Das Versprechen remplit apparemment toutes ces conditions. Une fillette a été assassinée dans un petit village des environs de Zurich; le commissaire Matthaï mène l'enquête; au dernier chapitre est révélée l'identité du maniaque criminel. Le dérapage, par rapport au modèle du récit de détection, se produit avec les modalités de découverte de la vérité. La désignation du coupable n'est pas le fruit de la raison (du détective), mais simplement celui du hasard - infraction caractérisée à la règle $n^{\circ} 5$ de Van Dine, qui exige que « le coupable soit découvert grâce à des déductions logiques et non pas par hasard ou grâce à des aveux non motivés $»^{10}$. C'est au terme d'un incroyable concours de circonstances - une vieille dame mourante qui, sur son lit d'hôpital, des années après les faits, confesse les turpitudes de son défunt mari - que l'identité du meurtrier est révélée au lecteur et non pas, justement, au détective, au représentant patenté de la raison à l'intérieur du récit. Non pas que la raison ait été véritablement mise en échec - les déductions du commissaire Matthaï étaient justes, le piège qu'il avait tendu eût parfaitement fonctionné si le criminel n'avait eu, avant d'arriver, un accident de la route ; mais son représentant, celui « qui voulait à toutes forces que la réalité répondît à la justesse de son calcul » ne peut pas lui-même recevoir la preuve qu'il ne se trompe pas. Le cours du monde n'est pas nécessairement « absurde » (il faut manipuler avec précaution cette catégorie, souvent requise à propos de Dürrenmatt, en particulier de son théâtre), il est paradoxal : il y a un sens, mais toujours irrévocablement décalé. Cette vision entraîne une réévaluation complète du personnage du détective, devenu une sorte de Don Quichotte obstiné et grotesque de la vérité, à l'image du commissaire Bärlach.

13 Avec Justiz, F. Dürrenmatt falsifie de manière encore plus radicale le code du récit de détection en inversant le sens, la fonction même de l'enquête. Il y a flagrant délit: en plein jour, à Zurich (?) au milieu de la foule d'un restaurant réputé, le Dr Isaak Kohler, homme politique en vue, abat de sang froid un des clients. Il est arrêté, jugé, condamné à vingt ans de réclusion. C'est depuis sa cellule qu'il mande un jeune avocat désoeuvré, Spät, auquel il confie, en une sorte de pacte diabolique, la mission de « recommencer l'enquête, en partant de l'hypothèse qu'il n'est pas le meurtrier $»^{11}$. Au lieu, donc, d'être une démarche au service de la "réalité des faits", voici une enquête dirigée contre 
ceux-ci : «Voyez vous, M. Spät, souvent, la nuit, lorsque je regarde les étoiles à travers les barreaux de ma cellule, je me demande à quoi ressemblerait la réalité, si ce n'était pas moi, mais un autre qui était le meurtrier... $»^{12}$. Et effectivement, contre toute évidence, au mépris des faits eux-mêmes, l'hypothèse spécieuse va être finalement reconnue comme plus vraie que la réalité : après que l'un des proches de Kohler s'est suicidé (fournissant ainsi un coupable idéal), le procès est révisé et l'acquittement prononcé. Cette perversion de la fonction de l'enquête - chargée ici d'avérer le faux fournit naturellement l'occasion d'une satire féroce de l'institution judiciaire et de la société helvétique (et il n'est pas indifférent que le personnage assumant le rôle de l'enquêteur appartienne non plus directement au personnel de la police, mais à celui de la justice: il lui revient en principe, non seulement de dire la vérité, mais aussi le Droit). Au-delà de cette charge se profile, comme toujours chez F. Dürrenmatt, un enjeu métaphysique, développé au dernier chapitre par le narrateur lui-même, dépositaire du manuscrit de Spät et de l'ultime «Vérité » des événements (Kohler, depuis le début, a mis en scène une "partie de billard » : pour venger sa fille Hélène violée par une clique de dépravés, il a joué " par la bande », touchant sa première victime pour atteindre la seconde) :

Und Gott? Denken wir ihn, hat er anders gehandelt als Dr h.c. Isaak Kohler? Hatte Spät nicht die Freiheit, den Auftrag abzuweisen, einen Mörder zu suchen, den es nicht gab? Musste er der denn nicht einen Mörder finden, den es nicht gab, so wie der Mensch, als er die Frucht des Baumes der Erkenntnis des Guten und des Bösen ass, den Gott finden musste, den es nicht gab, den Teufel? Ist dieser nicht die Fiktion Gottes, um seine missraten Schöpfung zu rechtfertigen? Wer ist der Schuldige ? Jener, der den Auftrag gibt, oder jener, der ihn annimmt? ${ }^{13}$

Bien loin du roman-jeu d'une «murder party», le récit de détection, révisé par F. Dürrenmatt, débouche sur la question ultime, ressassée dans toute son œuvre, autant romanesque que théatrale, de l'existence du mal. Plus grotesque encore que le commissaire Matthaï, à l'antipode lui-même de la superbe infaillibilité d'un Dupin ou d'un Holmes, l'enquêteur - «Spät», celui qui arrive (trop) tard - figure la condition trop humaine qui veut que "nous périssions de la liberté que nous autorisons et nous accordons à nous-mêmes » ${ }^{14}$.

Comme pour Justiz, le récit de Max Frisch Blaubart est centré non pas directement sur l'information policière, mais sur la procédure judiciaire. Ce décalage n'abolit pas, mais repousse dans les marges de la narration l'intrigue criminelle : un meurtre a été commis (Rosalinde Z., " demi-mondaine » de luxe, a été retrouvée étranglée dans son appartement) ; un suspect est arrêté (le Dr Félix Schaad, ex-époux de la victime) ; il est jugé, «acquitté faute de preuve »; à la dernière page, l'identité du vrai coupable est révélée (un étudiant grec qui, comme « client » de la jeune femme, possédait la clef de l'appartement, dans lequel il a trouvé l'arme du crime, une cravate ayant appartenu effectivement à Schaad). Cette intrigue permet sans doute au récit de fonctionner, mais n'en constitue pas l'objet premier, qui est la relation minutieuse du procès de Félix Schaad (dont le lecteur connaît d'ailleurs l'issue dès les premières pages). Les débats du procès, avec toutes les dépositions des témoins cités par le tribunal, ont ceci d'une enquête policière qu'ils correspondent à une recherche de la vérité (mais la finalité n'est pas tout à fait la même : on ne répond pas directement à la question "Qui est le criminel? », mais "Qui est ce criminel ?»). Le récit de M. Frisch démonte le rituel judiciaire de la quête de la vérité - « toute la vérité, rien que la vérité »-, en démontre l'inanité. Il ne s'agit pas de sa part, à la différence de F. Dürrenmatt dans Justiz, de 
polémiquer contre l'institution elle-même et de fustiger, à travers elle, l'hypocrisie sociale. Le tribunal est ici parfaitement honnête et rigoureux dans sa démarche, les témoins cités sont sincères. C'est la procédure de la quête qui est inadéquate. Elle dérive vers une mise en scène du vertige de l'identité, obsédant, comme on sait, dans toute l'œuvre de M. Frisch. La culpabilité est encore un rôle à endosser, un masque à porter. Bien que le tribunal ait prononcé l'acquittement, le docteur Schaad se comporte encore en coupable. L'arrestation du véritable meurtrier, à la fin, n'y change rien. S'il se sent coupable - au point de retourner dans son village natal pour avouer le meurtre à la police locale -, c'est donc qu'il l'est quelque part. Il faudrait ne pas être obligé de se choisir, d'arrêter une identité : Félix Schaad est bien le parent de Stiller.

Comparé aux récits de F. Dürrenmatt et M. Frisch, Eine gefährliche Begegnung d'E. Jünger colle plus près au schéma classique du récit de détection (le texte lui-même multiplie les références au roman policier anglais, au personnage de Sherlock Holmes ${ }^{15}$ ). Le crime : Paris, septembre 1888; une danseuse est assassinée à La cloche d'or, maison de rendez-vous discrète et fort bien fréquentée sise derrière La Madeleine; scandale dans la bonne société «Belle Epoque " parisienne, émoustillée par les articles de presse sur Jack the Ripper... L'histoire prend un tour original avec l'entrée en scène du détectivepolicier, un certain inspecteur Dobrowsky, qui aborde la criminalité en esthète, prêt à reconnaître les véritables « artistes », réfutant « toute théorie sociale et économique du crime » :

Die Theorien über das Verbrechen waren unzureichend, weil sie sich auf die Symptome beschränkten, nämlich auf den Schaden, der in der Gesellschaft entsteht. Es ist aber ein Unterschied zwischen diesem nachweisbaren Schaden und der verbrecherischen Potenz. Diese Potenz ist im Bösen beheimatet; es ist möglich, dass sie nie ihre Höhle verlässt und als Verbrechen sichtbar wird. Doch wie nach Heraklit die verborgene Harmonie grösser ist als die sichtbare, so ist auch diese geheime Residenz des Bösen gefährlicher als die Summe aller Untaten, die wir wahrnehmen. Das erfasst kein Gericht ${ }^{16}$.

17 Le policier de Jünger ne se veut le représentant ni de la société, ni de la morale ( « il faut faire des concessions à l'anarchie ; qui voudrait tout réprimer boucherait les soupapes de sûreté ${ }^{17}$ ) : il joue aux échecs, visite une galerie. Il n'en assume pas moins son rôle l'inspecteur Dobrowsky, grâce à ses capacités de déduction et, il le reconnaît, avec un peu de chance, résoud l'énigme du meurtre: il soupçonne un certain Monsieur de Kargané d'avoir assassiné la danseuse par méprise, la confondant avec sa femme qui avait ce soir-là rendez-vous, au même étage, avec un jeune attaché d'ambassade nommé Gerhard ; apprenant que Kargané a provoqué son rival en duel, il se précipite sur les lieux, accuse Kargané de meurtre et voit celui-ci retourner alors son arme contre lui-même. Dira-t-on pour autant que l'ordre triomphe ? Dobrowsky se reconnaît « moins le mérite d'avoir élucidé une affaire que d'avoir évité beaucoup de bruit autour d'elle ${ }^{18}$; il s'apprête à classer le dossier sans suite, car «il n'y a rien à gagner à rapporter un gibier dont personne ne veut [...] c'est un travail pour détectives privés ou journalistes payés pour cela ». Paradoxe d'un personnage de policier gardien lucide et détaché non pas d'un ordre, mais d'une apparence sociale.

Chacun, donc - Dürrenmatt, Frisch, Jünger - investit et travestit évidemment à sa manière le code narratif du récit de détection. Derrière les préoccupations singulières, on peut reconnaître les points clés à partir desquels s'opèrent les différentes dérives. Quels sont en effet les présupposés de tout récit de détection, ses conditions de possibilités narratives/idéologiques? Elles se résumeraient ainsi: 1) il y a, sur le 
monde, une " vérité ", fût-elle partielle ; 2) cette vérité est accessible et dicible ; 3) cette vérité est bonne à dire. Les auteurs des textes que nous avons considérés semblent paradoxalement revendiquer et en même temps révoquer en doute ces postulats. Plus exactement : ils voudraient encore y souscrire, malgré tout, contre tout. Le modèle du récit de détection serait comme l'utopie du Récit.

\section{Crimes incorrigibles}

Esquissons maintenant le cahier des charges de «l'histoire criminelle»:

1) Un crime est commis, sans caractère proprement énigmatique (Qui ? Comment ?) ; une seule question demeure éventuellement en suspens: qu'est-ce qui a poussé le criminel au crime ? - question qui va au-delà de la recherche d'un simple « mobile ».

2) Acteur principal de l'intrigue, qui focalise l'ensemble de la narration : le criminel (s'il arrive qu'un détective reçoive en tout ou partie cette fonction, celui-ci est alors une sorte de " criminel à l'envers " ${ }^{19}$, recourant plus à la force qu'à la logique).

3) Ressort de l'intrigue : comment le criminel «vit» son crime, avant et /ou après (donc, dans ce dernier cas, la plupart du temps une fuite-poursuite), ce qui implique en principe une narration chronologique (reposant sur une homologie entre la succession « réelle » des événements de l'histoire et l'ordre dans lequel ils sont narrés).

4) La conclusion du récit voit ou ne voit pas la restauration de l'ordre.

Le récit de H. Boll, Die verlorene Ehre der Katharina Blum s'ouvre sur une prolepse. Le narrateur raconte "à l'avance ", en quelques phrases lapidaires, ce qu'il appelle «les faits bruts » au double sens du terme : le mercredi 20 février 1974, vers 19 h 04, une jeune femme de 27 ans, Katharina Blum, sonne à la porte du commissaire de la brigade criminelle de Cologne et lui déclare avoir le jour même abattu chez elle de plusieurs coups de feu un journaliste. Cette anachronie narrative sert à une mise à distance du code de l'histoire criminelle, renforcée par une méfiance stylistique :

Es soll hier nicht so viel von Blut gesprochen werden, denn nur notwendige Niveauunterschiede sollen als unvermeidlich gelten, und deshalb wird hiermit aufs Fernsehen und aufs Kino verwiesen, auf Grusi- und Musicals einschlägiger Art; wenn hier etwas fliessen soll, dann nicht Blut ${ }^{20}$.

21 L'unique objet du récit, dans ces conditions (puisque l'identité du criminel est affichée, sa méthode avouée, sa poursuite inutile) est de répondre à la question de savoir ce qui l'a poussé au crime. Mais il s'agit en fait - c'est là tout le truquage opéré par H. Boll d'une fausse question, en ce sens où celui qui la pose (le narrateur) connaît déjà la réponse, affirmée péremptoirement dans le sous-titre - «Comment peut naître la violence et où elle peut conduire » - ainsi que dans l'exergue - «Si certaines pratiques journalistiques décrites dans ces pages offrent des ressemblances avec celles du journal Bild, ces ressemblances ne sont ni intentionnelles ni fortuites, mais tout bonnement inévitables ».

A l'image de la structure narrative de Die verlorene Ehre der Katharina Blum, le roman d'A. Muschg Albissers Grund se débarrasse d'entrée de jeu, en quelques lignes présentées sous forme de compte rendu, de toute l'information sur le contenu «criminel » de l'histoire : un jour d'été 1973, un certain Peter Albisser, professeur d'anglais au lycée de Zurich, a tenté d'assassiner de plusieurs coups de feu, à son domicile, un résidant étranger du nom de Zerutt, graphologue de son état ; grièvement blessé, la victime a été transportée à l'hôpital, où ses jours ne sont plus en danger, tandis que le meurtrier 
s'est rendu sans résistance à la police; Albisser, lors de son premier interrogatoire, a immédiatement reconnu les faits, mais s'est obstinément refusé à donner toute explication de son geste. Le lecteur se voit donc invité, comme chez Boll, à suivre une reconstitution des raisons du criminel. La différence, cependant, tient au fait que ne s'interpose ici aucun narrateur omniscient (même déguisé) qui serait le dépositaire et le porte-parole de la juste interprétation. Le crime est (ou plutôt serait) toujours un symptôme, mais ni l'auteur, ni le lecteur ne savent d'avance exactement de quoi : il s'inscrit dans une dialectique complexe du désir et de la réalité, dont l'enjeu plus ou moins conscient est le meurtre du père.

Par rapport au crime de Katharina Blum et d'Albisser, celui que commet le personnage principal de Die Angst des Tormanns beim Elfmeter, de P. Handke, pourrait être qualifié de plus "simplement compliqué». Le monteur Joseph Bloch, qui a été congédié de son travail - du moins le croit-il - erre dans la ville, rencontre une caissière de cinéma qu'il étrangle au matin chez elle - « il avait immédiatement serré si fort qu'elle n'avait pas eu le temps de croire à une farce ". Dans le texte lui-même, le meurtre ne reçoit aucun sens téléologique, comme chez $\mathrm{H}$. Boll, ne fait pas même l'objet d'un travail herméneutique, comme chez A. Muschg. Il est révélateur que, dans l'ordre de la narration, il ne soit pas mis en relief en position initiale (comme chez H. Boll et A. Muschg), mais au contraire décalé, banalisé dans une succession d'actions non spectaculaires, quotidiennes : comme si le meurtre ne constituait plus, a priori, un événement inouï, susceptible d'enclencher à lui seul un récit. P. Handke laisse son lecteur entièrement libre de son interprétation, sans autre support que la contiguïté des séquences narratives. Bloch tue la jeune fille étendue à ses côtés juste après qu'elle lui a demandé "s'il allait au travail aujourd'hui » et qu'il s'est auparavant aperçu " qu'elle parlait de choses qu'il venait à peine d'évoquer comme si déjà elles lui étaient propres, alors que lui, chaque fois qu'il reprenait une chose dont elle venait de parler [...] maintenait une distance, comme s'il craignait de s'approprier ce qui la concernait ». Thème handkéen du terrorisme du langage. Le meurtre, dans cette perspective, n'est pas seulement un symptôme. Il peut être une manière de reconquérir le sens. La fuite, la poursuite - nécessairement escamotées, chez Boll comme chez Muschg - retrouvent ici une fonction. A partir du meurtre, Joseph Bloch va être contraint de déchiffrer le monde " devenu un espace de soupçon ", où tous les signes deviennent des indices «qui font allusion à lui ». L'attention au monde ne se reconquiert, tout au moins dans ce roman-ci de P. Handke, que dans la menace et l'angoisse. La fin de l'histoire, avec l'image du penalty arrêté et de la frontière qui court non loin du village où Bloch s'est réfugié, laisse éventuellement entrevoir un dépassement : au-delà vont commencer, à partir de 1970, les « récits de voyage » du romancier.

Comparable à Die Angst des Tormanns beim Elfmeter serait le roman de D. Wellershoff Die Schattengrenze, dans la mesure où le personnage du criminel, qui focalise le récit, exemplifie ici aussi une relation "pathologique» aux autres et au monde (il est d'ailleurs notable que les deux histoires se terminent non loin d'une frontière géographique, mais qui est aussi celle entre «normalité » et schizophrénie). La différence est dans le rapport inverse aux signes : tandis que Joseph Bloch recueille tout ce par quoi le monde pourrait lui «faire signe " après le meurtre, le personnage de D. Wellershoff (coupable d'actes délictueux et non véritablement criminels: malversations financières, trafics de voitures volées etc) s'efforce au contraire en vain d'effacer ses propres traces. On retrouve la différence aperçue au niveau de la réflexion poétique des deux romanciers: le monde n'a d'autre sens, chez P. Handke, que par 
rapport au sujet tandis que chez D. Wellershoff, le sujet se construit (ou ne parvient pas à se construire) par rapport au monde.

Einladung an alle, le prochain roman « néo-policier » de D. Wellershoff, est encore centré sur une fuite-poursuite, mais comprise cette fois-ci de manière tout à fait concrète, alors qu'elle restait théorique dans Die Schattengrenze (le lecteur ne sait pas si la police recherche vraiment le héros suspect, ou s'il se l'imagine seulement). On suit ici, relatée avec une précision documentaire, la traque d'un « criminel », à travers la province de Basse-Saxe, par la police d'Osnabrück. La facture originale du roman tient au changement de " points de vue » qu'il installe: d'un côté l'individu isolé, marginal, de l'autre non pas un adversaire précis, personnel (comme le policier ou le «privé » du roman noir), mais toute une institution sociale, dont le récit démonte les rouages et mécanismes. C'est d'ailleurs cette institution qui fait de Findeisen un criminel: le vagabond qu'il était n'avait commis que des larcins lorsque, sur le point d'être pris, il tua un gendarme. On est très proche, finalement, de la «morale » de Die verlorene Ehre der Katharina Blum. Comme H. Boll, D. Wellershoff démonte et dénonce les mécanismes de l'hystérie collective. Mais il ne s'agit pas d'une leçon donnée a priori et dont le récit serait l'illustration. C'est le texte lui-même qui, ici, dans sa facture, induit une critique idéologique.

$\mathrm{Au}$ terme de nos lectures, qui ont permis de constater comment chaque romancier, comme pour le récit de détection, parvient à détourner à son profit le code narratif de l'histoire criminelle, nous pouvons cerner le point sur lequel se jouent les diverses contrefaçons repérées. Quel est le présupposé intangible de toute histoire criminelle? Que le crime, précisément, «fait une histoire». Dans les textes que nous avons lus, la fiction criminelle vient effectivement garantir une sorte de " minimum narratif ", offrir comme un "noyau dur» du romanesque ${ }^{21}$, à partir duquel il (re)devient possible de dire, de lire le monde. Chacun, évidemment, selon ses convictions : depuis l'affirmation péremptoire d'un sens univoque, comme chez Boll, au simple postulat d'une lente conquête et (re)construction d'un sens possible, comme chez Handke. Récit de détection ou histoire criminelle, le genre policier paraît en tout cas confirmé dans sa fonction d'ultime et paradoxal recours d'une écriture "réaliste», décidée à risquer encore une mise en forme esthétique du monde :

Wider Erwarten erscheint der Kriminalroman so als eine der offensten Formen der heutigen Literatur [...] Überdies handelt es sich beim Kriminalroman um etwas, was so viele Kritiker der modernen Literatur vermissen: nämlich um legitimen Lesestoff für alle... (Helmut Heissenbüttel) ${ }^{22}$.

\section{NOTES}

1. - Friedrich Dürrenmatt, Das Versprechen, München, 1978, p. 9-10.

2. - Cité d'après Theorie des Kriminalromans, Reclam, Stuttgart, 1974, p. 35.

3. - Ibid., p. 50.

4. - «Alle realistischen Elemente, seien sie psychologischer, wirtschaftlicher oder sozialer Art, müssen von vornherein so eingerichtet sein, dass sie zu verschlüsselbaren wie auch auflösbaren 
Musterspielen zusammengefügt werden können. Es ergeben sich zwei Stilisierungsmöglichkeiten, die ins Abnorme und die Reduktion. In der Abnormität finden sich die Nachkommen von Poes Orang-Utan. Hier geht der Kriminalroman über in den Thriller, die Gruselgeschichte. Die Reduktion führt zu einer weiteren Einsicht. Es zeigt sich, dass es dem Kriminalroman gerade auf das nicht ankommt, auf was es dem Roman der sogenannten seriösen Literatur ankommt: auf Menschendarstellung und auf die Ergründung menschlicher Motive in Reflexion und Haltung [...]. Der Kriminalroman ist eine Exempelgeschichte, die nach einem bestimmten Schema etwas einübt» (Helmut Heissenbüttel, «Spielregeln des Kriminalromans», in Aufsätze zur Literatur, München, 1972, p. 93).

5. - Peter Handke, «Über meinen Roman Der Hausierer», in Dichten und Trachten, 1967.

6. - Cf. Christoph F. Lorenz, «Dem Täter auf der Spur. Dieter Wellershoffs theoretische und literarische Beschäftigung mit dem Detektiv- und Kriminalroman».

7. - Dieter Wellershoff, «Fiktion und Praxis», in Literatur und Veränderung, Köln, 1969, p. 22-23.

8. - Dieter Wellershoff, «Vorübergehende Entwirklichung. Zur Theorie des Kriminalromans», in Von der Moral erwischt. Aufsätze zur Trivialliteratur, Frankfurt/Main, 1983, p. 81.

9. - Richard Alewyn, «Anatomie des Detektivsromans», in Der Kriminalroman. Zur Theorie und Geschichte einer Gattung (hrsg. v. J. Vogt), München, 1971, p. 372-404.

10. - Op. cit., p. 32.

11. - Friedrich Dürrenmatt, Justiz, Zürich, 1985, p. 86.

12. - Ibid., p. 87.

13. - Ibid., p. 368-369.

14. - Ibid., p. 369.

15. - Ernst Jünger, Eine gefährliche Begegnung, Stuttgart, 1985, p. 105, 162, etc.

16. - Ibid., p. 91.

17. - Ibid., p. 92.

18. - Ibid., p. 162.

19. - Boileau-Narcejac, Le roman policier, P.U.F., Paris, 1975, p. 75.

20. - H. Boll, Die verlorene Ehre der Katharina Blum, Köln, 1974, p. 13.

21. - Cf. Yves Reuter, Le roman policier et ses personnages, P.U.V., Saint-Denis, 1989, p. 220. On retiendra, dans cet ouvrage, la contribution d'Alain Montandon, « L'Angoisse du penalty : Handke et le policier ».

22. - Op. cit., p. 103-104.

\section{RÉSUMÉS}

Toute une famille d'ouvrages, dus aux plus grands noms de la littérature allemande contemporaine - H. Boll, F. Dürrenmatt, M. Frisch, P. Handke, E. Jünger, A. Muschg, D. Wellershoff etc. - ont été évalués par la critique et également, dans la plupart des cas, désignés explicitement par leurs auteurs eux-mêmes comme des fictions néoolicières. Il appert que les multiples manipulations, subversions, falsifications de ce code narratif - que l'on se réfère au schéma du récit de détection à celui de l'histoire criminelle -, sont mobilisées au profit d'une sorte d'utopie d'écriture "mimétique ", c'est-à-dire encore capable de lire, de dire la réalité, quelle que soit l'idée que l'on se fait de celle-ci, quels que soient les risques de l'entreprise. Loin 
de représenter une concession à la littérature de consommation, les contrefaçons de fictions policières apparaissent comme un ultime recours du romanesque.

Eine ganze Reihe von Texten, von den grössten zeitgenössischen deutschsprachigen Autoren H. Boll, F. Dürrenmatt, M. Frisch, P. Handke, E. Jünger, A. Muschg, D. Wellershoff... - sind von der Kritik und in den meisten Fällen auch explizit von ihren Verfassern selbst als NeoKriminalliteratur bezeichnet worden. Zweck der vorliegenden Analyse ist, einige der Umschreibungstechniken zwischen Belletristik und Kriminalliteratur aufzuzeigen. Es wird dabei deutlich, dass viele Manipulationen, Abwandlungen und Verfälschungen dieses Erzählcodes, sei es in bezug auf das Schema der Detektivgeschichte oder auf das der Kriminalerzählung, in Gang gesetzt werden im Dienste einer Art von Utopie der mimetischen Schreibweise, d.h. einer Schreibweise, die noch fähig ist, die Wirklichkeit zu entziffern und wiederzugeben, welche Vorstellung man auch immer von dieser Realität hat und welche Risiken man dabei auch eingeht. Weit davon entfernt, ein Zugeständnis an die Trivialliteratur darzustellen, erscheint die nachgemachte Kriminalliteratur als ein letzter Zufluchtsort der Gattung Roman.

\section{AUTEUR}

JEAN-JACQUES POLLET

Université Charles-de-Gaulle - Lille III 